\title{
Strip Line Device
}

National Cancer Institute

\section{Source}

National Cancer Institute. Strip Line Device. NCI Thesaurus. Code C50194.

A conductor cable designed as a thin, flexible ribbon of metals separated by an insulating layer. 